\title{
Passive flutter suppression using a nonlinear tuned vibration absorber
}

\author{
G. Habib, G. Kerschen \\ Space Structures and Systems Laboratory (S3L), Structural Dynamics Research Group \\ Department of Aerospace and Mechanical Engineering, University of Liège, Belgium
}

\begin{abstract}
Limit cycle oscillations (LCOs) occur in many mechanical systems and they are often a source of danger. The addition of a relatively small mass to the host system, attached through a linear spring and a damper (linear tuned vibration absorber, LTVA), significantly improves its stability. The use of a purely nonlinear spring in the absorber (nonlinear energy sink, NES) increases the frequency bandwidth of the absorber, reduce LCO amplitude and avoid subcritical bifurcation at the loss of stability. Recently, a nonlinear tuned vibration absorber (NLTVA) has been proposed, whose restoring force is tailored according to the functional form of the nonlinearity of the primary system. The NLTVA is designed to exploit the positive features of both the LTVA and the NES. In this paper we show that the NLTVA can compensate the detrimental effect of the nonlinearities of the primary system. In other words, the coupled system exhibits a linear-like dynamics, similar to the same system without structural nonlinearities. Considering a Van der Pol - Duffing oscillator as primary system, a closed-form solution for the local compensation of nonlinearities is obtained analytically. Numerical continuation techniques show that the compensation is valid also for large amplitudes of the response.
\end{abstract}

Keywords: limit cycle oscillations, vibration absorber, nonlinearity synthesis, stability analysis, bifurcation analysis.

\section{INTRODUCTION}

Limit cycle oscillations (LCOs) are encountered in a number of real engineering applications including aircraft ${ }^{[1,2]}$, machine tools ${ }^{[3,4]}$, automotive disk brakes ${ }^{[5,6]}$ and oil-drilling rigs ${ }^{[7]}$. LCOs often limit the performance and can also endanger the safety of operation ${ }^{[8]}$.

Active control strategies have been proposed as a means of counteracting LCOs ${ }^{[9-11]}$. These references have shown that active control can be used to raise the threshold above which LCOs occur. However, active control is also limited by its requirements in terms of energy or space for actuators. Furthermore, delay in the feedback loop can generate unexpected instabilities ${ }^{[12,13]}$ whereas saturation of the actuators can limit the robustness of stability ${ }^{[14]}$.

Passive vibration absorbers are another alternative for mitigating undesired LCOs. Specifically, the linear tuned vibration absorber (LTVA), which comprises a small mass attached to the host system through a damper and a spring, has been widely studied in the literature ${ }^{[15-21]}$. In most of these works, the system under investigation is the classical Van der Pol (VdP) oscillator. References $[17,18]$ provide simple rules to properly tune the LTVA parameters, while references ${ }^{[20,21]}$ study the post-bifurcation behavior of the coupled system. In ${ }^{[22]}$, a nonlinear damping element was added in parallel with the LTVA to decrease the maximum LCO amplitude. Other nonlinear vibration absorbers, including the autoparametric vibration absorber ${ }^{[23,24]}$, the nonlinear energy sink ${ }^{[25-27]}$ and the hysteretic tuned vibration absorber ${ }^{[28]}$, have also been considered to increase the effectiveness of vibration attenuation.

The main idea of this study is to utilize the nonlinear tuned vibration absorber (NLTVA) for LCO suppression. This absorber, first introduced in ${ }^{[29]}$, possesses a linear spring and a nonlinear spring whose mathematical form is determined according to the 
nonlinearity in the host system. Following references ${ }^{[15-21]}$, the linear spring coefficient is determined to maximize the stable region of the trivial solution of the host system. Subsequently, the nonlinear spring is designed to ensure supercritical behavior and to mitigate the LCOs in the postcritical range. A fundamental result of this paper is that, if properly designed, the linear and nonlinear springs of the NLTVA can complement each other giving rise to a very effective LCO suppression and management strategy. The example that will serve to validate the proposed developments is the Van der Pol-Duffing (VdPD) oscillator ${ }^{[30]}$, which is a paradigmatic model for the description of self-excited oscillations. The bifurcation behavior of the VdPD oscillator was studied in ${ }^{[31,32]}$ whereas its stabilization using active control was proposed in ${ }^{[33-35]}$.

The paper is organized as follows. Section 2 introduces the three design objectives pursued in this paper. In Section 3, optimal values for the linear parameters of the NLTVA are determined using stability analysis of the coupled system. Section 4 investigates the bifurcations occurring at the loss of stability and proposes an analytical tuning rule for the nonlinear coefficient of the NLTVA. In Section 5, the reduction of the LCO amplitude in the postcritical range is discussed. Local and global analyses are carried out using normal form theory and the MATCONT software, respectively. Finally, conclusions are drawn in Section 6.

\section{PROBLEM FORMULATION}

The primary system considered throughout this work is the Van der Pol-Duffing (VdPD) oscillator:

$$
m_{1} q_{1}^{\prime \prime}+c_{1}\left(q_{1}^{2}-1\right) q_{1}^{\prime}+k_{1} q_{1}+k_{n l 1} q_{1}^{3}=0
$$

where $m_{1}, c_{1}, k_{1}$ and $k_{n l 1}$ are the oscillator's mass, damping and the coefficients of the linear and cubic springs, respectively. For instance, for an in-flow wing, the terms $c_{1}\left(q_{1}^{2}-1\right) q_{1}^{\prime}$ and $k_{n l 1} q_{1}^{3}$ would model the fluid-structure interaction and the structural nonlinearity, respectively. The trivial equilibrium point of the system loses stability when $\mu_{1}=c_{1} / 2 \sqrt{k_{1} m_{1}}=0$. Loss of stability occurs through either a supercritical Hopf bifurcation or a subcritical Hopf bifurcation. This latter scenario is dangerous, because stable large-amplitude LCOs can co-exist with the stable equilibrium point ${ }^{[36]}$.

The objective of the present study is to mitigate, or even completely eliminate, the LCOs of the VdPD oscillator through the attachment of a fully passive nonlinear vibration absorber, termed the NLTVA ${ }^{[29]}$. One salient feature of the NLTVA compared to existing nonlinear absorbers is that the absorber's load-deflection curve is not imposed a priori, but it is rather synthesized according to the nonlinear restoring force of the primary system. The equations of motion of the coupled VdPD and NLTVA system are:

$$
\begin{aligned}
& m_{1} q_{1}^{\prime \prime}+c_{1}\left(q_{1}^{2}-1\right) q_{1}^{\prime}+k_{1} q_{1}+k_{n l 1} q_{1}^{3}+c_{2}\left(q_{1}^{\prime}-q_{2}^{\prime}\right)+g\left(q_{1}-q_{2}\right)=0 \\
& m_{2} q_{2}^{\prime \prime}+c_{2}\left(q_{2}^{\prime}-q_{1}^{\prime}\right)-g\left(q_{1}-q_{2}\right)=0
\end{aligned}
$$

where $m_{2}$ and $c_{2}$ are the absorber's mass and viscous damping, respectively. The NLTVA is assumed to have a generic smooth elastic force $g\left(q_{1}-q_{2}\right)$ with $g(0)=0$.

The design problem is as follows. The mass ratio $\varepsilon=m_{2} / m_{1}$ (and, hence, the absorber mass) is prescribed by obvious practical constraints; $\varepsilon=0.05$ is considered in the numerical examples of this paper. The damping coefficient $c_{2}$ and the absorber's stiffness $g\left(q_{1}-q_{2}\right)$ should be determined so as to:

1. Maximize the stable region of the VdPD oscillator by displacing the Hopf bifurcation toward large positive values of $\mu_{1}$ (Fig. $1(\mathrm{a}))$

2. Avoid a catastrophic bifurcation scenario by transforming the potentially subcritical Hopf bifurcation of the VdPD into a supercritical Hopf bifurcation of the coupled system (Fig. 1(b));

3. Reduce the amplitude of the remaining LCOs (Fig. 1(c)).

These three design objectives are studied in detail in the next three sections.

\section{ELIMINATION OF LIMIT CYCLES THROUGH STABILITY ANALYSIS}

The first design objective is to stabilize the trivial solution of the VdPD oscillator for values of $\mu_{1}$ greater than 0 . Because the stability of an equilibrium point of a nonlinear system is governed only by the local underlying linear system, the NLTVA should 

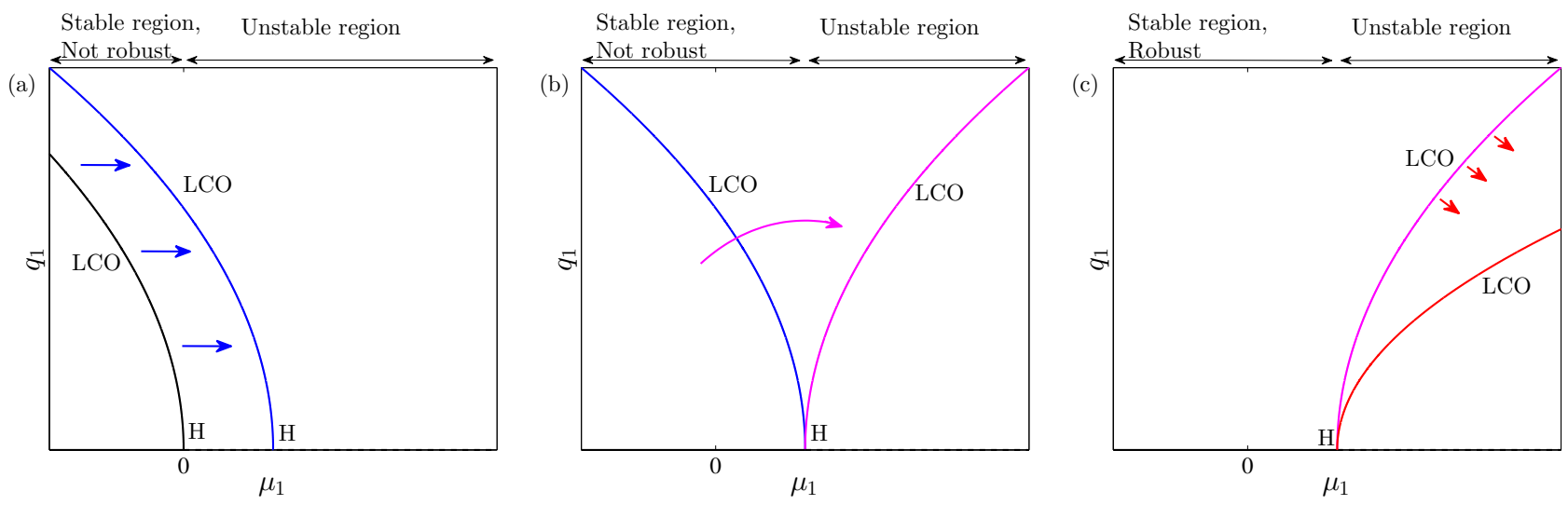

Figure 1: Subcritical VdPD oscillator with an attached NLTVA. (a) Enlargement of the stable region of the equilibrium point of the VdPD oscillator; (b) transformation of the subcritical Hopf bifurcation into a supercritical Hopf bifurcation; (c) reduction of the amplitude of the remaining LCOs. $H$ stands for Hopf bifurcation.

comprise a linear spring for increased flexibility, i.e., $g\left(q_{1}-q_{2}\right)=k_{2}\left(q_{1}-q_{2}\right)+g_{n l}\left(q_{1}-q_{2}\right)$. The system of interest for the stability analysis is therefore

$$
\begin{aligned}
& m_{1} q_{1}^{\prime \prime}-c_{1} q_{1}^{\prime}+k_{1} q_{1}+c_{2}\left(q_{1}^{\prime}-q_{2}^{\prime}\right)+k_{2}\left(q_{1}-q_{2}\right)=0 \\
& m_{2} q_{2}^{\prime \prime}+c_{2}\left(q_{2}^{\prime}-q_{1}^{\prime}\right)+k_{2}\left(q_{2}-q_{1}\right)=0
\end{aligned}
$$

Introducing the variables $\omega_{n 1}^{2}=k_{1} / m_{1}, \omega_{n 2}^{2}=k_{2} / m_{2}, \mu_{2}=c_{2} /\left(2 m_{2} \omega_{n 2}\right), \gamma=\omega_{n 2} / \omega_{n 1}$, the dimensionless time $\tau=t / \omega_{n 1}$ and the variable $q_{d}=q_{1}-q_{2}$, and recasting the equations in matrix form yield

$$
\left[\begin{array}{ll}
1 & 0 \\
0 & 1
\end{array}\right]\left[\begin{array}{l}
\ddot{q}_{1} \\
\ddot{q}_{d}
\end{array}\right]+\left[\begin{array}{cc}
-2 \mu_{1} & 2 \mu_{2} \gamma \varepsilon \\
-2 \mu_{1} & 2 \mu_{2} \gamma(1+\varepsilon)
\end{array}\right]\left[\begin{array}{l}
\dot{q}_{1} \\
\dot{q}_{d}
\end{array}\right]+\left[\begin{array}{cc}
1 & \gamma^{2} \varepsilon \\
1 & \gamma^{2}(1+\varepsilon)
\end{array}\right]\left[\begin{array}{l}
q_{1} \\
q_{d}
\end{array}\right]=\left[\begin{array}{l}
0 \\
0
\end{array}\right]
$$

or in compact form $\mathbf{M} \ddot{\mathbf{q}}+\mathbf{C} \dot{\mathbf{q}}+\mathbf{K q}=\mathbf{0}$. The dot indicates derivation with respect to the dimensionless time $\tau$.

As reported for a similar system in ${ }^{[20]}$, the trivial solution of Eq. (4) is asymptotically stable if and only if the roots of the characteristic polynomial $\operatorname{det}\left(z^{2} \mathbf{M} \ddot{\mathbf{q}}+z \mathbf{C} \dot{\mathbf{q}}+\mathbf{K q}\right)=0$ have negative real parts. Fig. 2(a) depicts the stability chart in the $\mu_{1}, \mu_{2}, \gamma$ space obtained from direct evaluation of the roots. The surface, which represents the stability boundary, peaks along the $\mu_{1}$ axis at point $C$, meaning that the trivial solution can no longer be stable beyond this point. Performing the stability analysis through Routh-Hurwitz criterion the coordinates of point $\mathrm{C}$ can be found analytically. These allow us to define the optimal values of $\mu_{2}$ and $\gamma$ to maximize the value of $\mu_{1}$ that gives stability, namely

$$
\mu_{2 \mathrm{opt}}=\frac{1}{2} \sqrt{\frac{\varepsilon}{1+\varepsilon}}, \quad \gamma_{\mathrm{opt}} \frac{1}{\sqrt{1+\varepsilon}}
$$

which gives $\mu_{1 \max }=\sqrt{\varepsilon} / 2$.

\section{ENFORCEMENT OF SUPERCRITICAL HOPF BIFURCATIONS THROUGH NORMAL FORM ANALYSIS}

The second design objective is to ensure the robustness of the trivial solution, i.e., no stable LCO can coexist with the stable equilibrium, as depicted in Fig. 1(b). Since supercritical Hopf bifurcations are sought in the coupled system, a detailed investigation of the bifurcations occurring at the loss of stability is the main focus of the present section. Because bifurcation characterization depends on the nonlinear coefficient of the NLTVA, this analysis will allow us to define the optimal value of this coefficient whereas the linear coefficients of the NLTVA should remain close to their optimal values (5), i.e., $\gamma=0.976$ and $\mu_{2}=0.109$ for $\varepsilon=0.05$.

Another key element that remains to be determined is the mathematical expression of the NLTVA's elastic force $g\left(q_{1}-q_{2}\right)$. A reasoning based upon local analysis is adopted herein. First, fractional-order terms are not considered, since they would be 

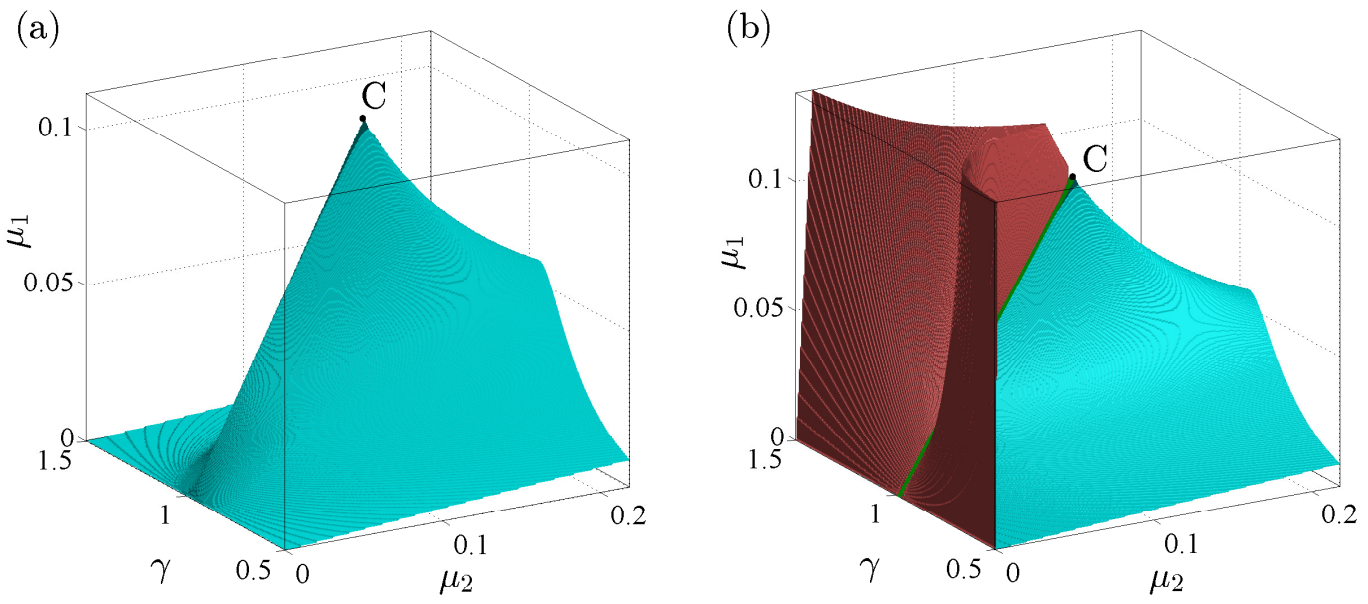

Figure 2: Stability chart in the $\mu_{1}, \mu_{2}, \gamma$ space for $\varepsilon=0.05$. The blue surface $(\mathbf{a}, \mathbf{b})$ indicates the stability boundary whereas the red surface (b) indicates the boundary of the region with four eigenvalues with positive real parts. The coupled system loses stability through double Hopf bifurcations along the green line (b).

transformed into terms of integer orders by the Taylor series expansion during bifurcation analysis. Terms of degree higher than 3 have negligible effects on the local dynamics; they would be neglected during the transformation into normal form. Although it is difficult to anticipate it without performing the analysis, quadratic terms give much less freedom for tuning the NLTVA compared to cubic terms. For these reasons, the nonlinear spring of the NLTVA is chosen to be cubic, and, hence, the coupled system writes

$$
\begin{aligned}
& m_{1} q_{1}^{\prime \prime}+c_{1}\left(q_{1}^{2}-1\right) q_{1}^{\prime}+k_{1} q_{1}+k_{n l 1} q_{1}^{3}+c_{2}\left(q_{1}^{\prime}-q_{2}^{\prime}\right)+k_{2}\left(q_{1}-q_{2}\right)+k_{n l 2}\left(q_{1}-q_{2}\right)^{3}=0 \\
& m_{2} q_{2}^{\prime \prime}+c_{2}\left(q_{2}^{\prime}-q_{1}^{\prime}\right)+k_{2}\left(q_{2}-q_{1}\right)+k_{n l 2}\left(q_{2}-q_{1}\right)^{3}=0
\end{aligned}
$$

Considering dimensionless coordinates, we transform the system into first-order differential equations

$$
\left[\begin{array}{c}
\dot{x}_{1} \\
\dot{x}_{2} \\
\dot{x}_{3} \\
\dot{x}_{4}
\end{array}\right]=\left[\begin{array}{cccc}
0 & 1 & 0 & 0 \\
-1 & 2 \mu_{1} & -\gamma^{2} \varepsilon & -2 \mu_{2} \gamma \varepsilon \\
0 & 0 & 0 & 1 \\
-1 & 2 \mu_{1} & -\gamma^{2}(1+\varepsilon) & -2 \mu_{2} \gamma(1+\varepsilon)
\end{array}\right]\left[\begin{array}{c}
x_{1} \\
x_{2} \\
x_{3} \\
x_{4}
\end{array}\right]+\left[\begin{array}{c}
0 \\
-2 \mu_{1} x_{1}^{2} x_{2}-\alpha_{3} x_{1}^{3}-\beta_{3} \varepsilon x_{3}^{3} \\
0 \\
-2 \mu_{1} x_{1}^{2} x_{2}-\alpha_{3} x_{1}^{3}-\beta_{3}(1+\varepsilon) x_{3}^{3}
\end{array}\right]
$$

or in compact form $\dot{\mathbf{x}}=\mathbf{W} \mathbf{x}+\mathbf{b}$. The variables $x_{1}=q_{1}, x_{2}=\dot{q}_{1}, x_{3}=q_{d}, x_{4}=\dot{q}_{d}, \alpha_{3}=k_{n l 1} / k_{1}$ and $\beta_{3}=k_{n l 2} /\left(k_{1} \varepsilon\right)$ have been introduced in these equations.

When stability is lost, one or two pairs of complex conjugate eigenvalues of $\mathbf{W}$ leave the left half plane, which corresponds to single or double Hopf bifurcation, respectively. As reported in ${ }^{[20]}$, a double Hopf bifurcation is likely to occur when $\gamma=\gamma_{\text {opt }}$ and $\mu_{2} \leq \mu_{\text {opt }}$. This assertion is confirmed in Fig. 2(b), which superposes the boundary of the region with four eigenvalues with positive real parts on the stability boundary of Fig. 2(a). If the coupled system loses stability along the green line where two pairs of complex conjugate eigenvalues have zero real part, a double Hopf bifurcation is encountered.

\subsection{Single Hopf bifurcation}

The analysis is first focused on the single Hopf bifurcation scenario for which $\mathbf{W}$ has a pair of complex conjugate eigenvalues with zero real part $\lambda_{1,2}=k_{1} \pm j \omega_{1}$ and two other eigenvalues $\lambda_{3}$ and $\lambda_{4}$ with negative real parts. Defining a transformation matrix through the eigenvectors of $\mathbf{W}$, we can decouple the linear part of the system. Then, applying a center manifold reduction it is possible to eliminate the variable not related to the bifurcation, reducing the dimension of the system without affecting the local dynamics. Performing then a transformation in complex form, a near identity transformation and a transformation in polar coordinates, we obtain the normal form of a Hopf bifurcation, i.e.

$$
\dot{r}=k_{1} r+\delta r^{3} \text {. }
$$

Eq. (8) has solutions $r_{0}=0$ and $r^{*}=\sqrt{-k_{1} / \delta}$. The coefficient $\delta$ can be expressed as a linear function of the nonlinear coefficients $\alpha_{3}$ and $\beta_{3}$ :

$$
\delta=\delta_{0}\left(\varepsilon, \gamma, \mu_{1}, \mu_{2}\right)+\delta_{\alpha_{3}}\left(\varepsilon, \gamma, \mu_{1}, \mu_{2}\right) \alpha_{3}+\delta_{\beta_{3}}\left(\varepsilon, \gamma, \mu_{1}, \mu_{2}\right) \beta_{3}
$$





Figure 3: Values of (a) $\delta_{0}$, (b) $\delta_{\alpha_{3}}$ and (c) $\delta_{\beta_{3}}$ along the stability boundary in the $\mu_{1}, \mu_{2}, \gamma$ space for $\varepsilon=0.05$. The color indicates the value of the corresponding coefficient. To facilitate the visualization, two different views of the same surface are given (top and bottom plots).
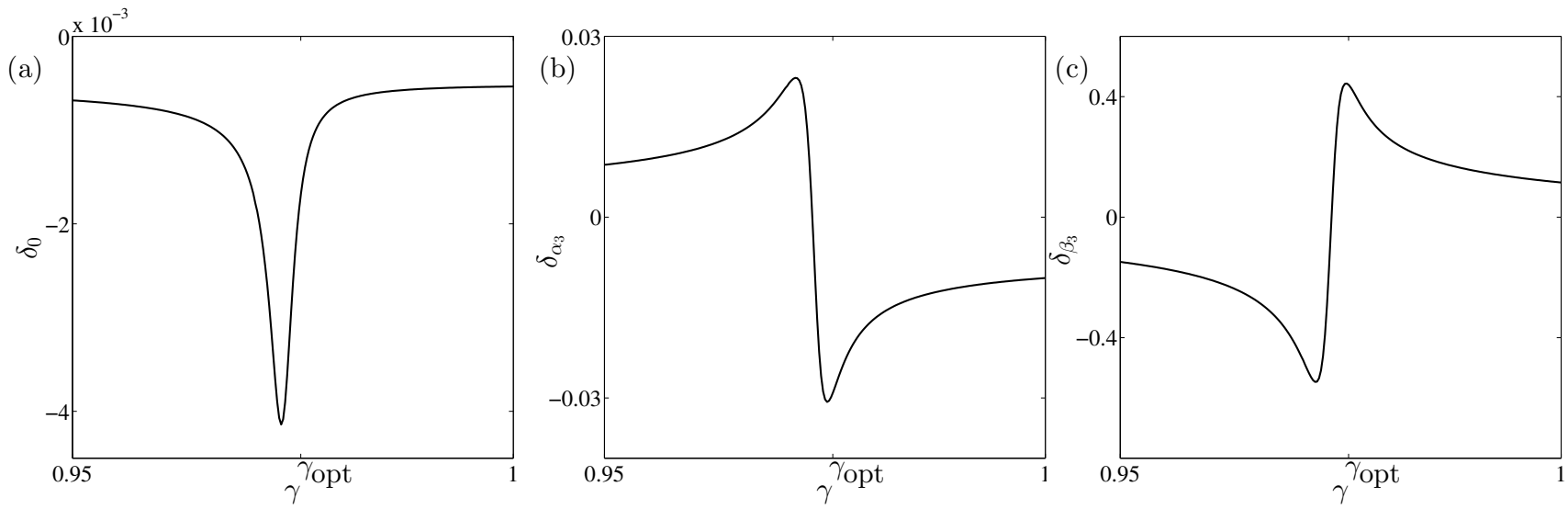

Figure 4: Values of (a) $\delta_{0}$, (b) $\delta_{\alpha_{3}}$ and (c) $\delta_{\beta_{3}}$ for $\mu_{2}=0.12$ and $\varepsilon=0.05$

where $\delta_{0}, \delta_{\alpha_{3}}$, and $\delta_{\beta_{3}}$ are defined analytically in the procedure just briefly described.

From Eq. (8), we see that the bifurcation is supercritical if $\delta<0$ and subcritical if $\delta>0$. Our objective should therefore be to design the nonlinear spring $\beta_{3}$ of the NLTVA to impose negative values of $\delta$. Ideally, this nonlinear tuning should be carried out in 


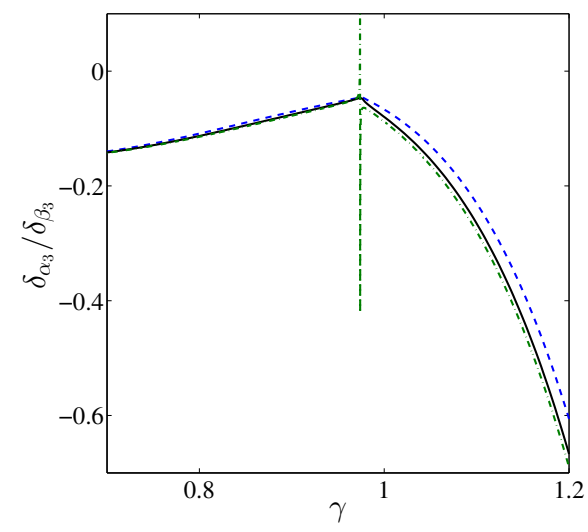

Figure 5: Ratio $\delta_{\alpha_{3}} / \delta_{\beta_{3}}$ as a function of $\gamma$ for fixed values of $\mu_{2}$ and $\varepsilon=0.05$. Dashed blue lines: $\mu_{2}=0.07$, solid black lines: $\mu_{2}=\mu_{2 \mathrm{opt}}=0.1091$, dash-dotted green lines: $\mu_{2}=0.12$. The dash-dotted green curve goes to infinity when $\delta_{\beta_{3}}=\mathbf{0}$; this is not a problem because $\delta_{\alpha_{3}}$ goes to 0 almost at the same time.

the vicinity of $\gamma_{\text {opt }}$ and $\mu_{2 \text { opt }}$ so as to maintain LCO onset at large values of $\mu_{1}$. Fig. 3 displays the values of the coefficients $\delta_{0}, \delta_{\alpha_{3}}$ and $\delta_{\beta_{3}}$ along the stability boundary in the $\mu_{1}, \mu_{2}, \gamma$ space. Fig. 4 considers a section of these plots for $\mu_{2}=1.1 \mu_{2 \mathrm{opt}}=0.12$ for which stability is lost through a single Hopf bifurcation.

The case of a LTVA (i.e., $\beta_{3}=0$ ) attached to the VdPD is investigated, and positive values of $\alpha_{3}$ are first considered. Because $\delta_{0}$ is always negative (this means that the system with no structural nonlinearity is always supercritical), our attention should be focused on the value of $\delta_{\alpha_{3}}$. Figs. 3(b) and 4(b) evidence a symmetric behavior for $\delta_{\alpha_{3}}$, i.e., it is positive (negative) below (above) $\gamma \approx \gamma_{\text {opt }}$. This uncertainty on the sign of $\delta_{\alpha_{3}}$ in the region of optimum tuning poses an important practical difficulty, because a supercritical bifurcation cannot confidently be enforced in this region. The solution to avoid a catastrophic bifurcation scenario is to detune the NLTVA toward larger values of $\gamma$, which guarantees negative values of $\delta_{\alpha_{3}}$. However, this detuning is associated with a significant decrease in the value of $\mu_{1 \max }$. For instance, considering $\gamma=1$ decreases $\mu_{1 \max }$ by approximately $30 \%$. Following a similar reasoning, we conclude that the NLTVA should be detuned toward smaller values of $\gamma$ for negative values of $\alpha_{3}$.

The NLTVA presents increased flexibility with respect to the LTVA, because $\beta_{3}$ represents an additional tuning parameter. However, Figs. 3(c) and 4(c) show that the sign of $\delta_{\beta_{3}}$ in the optimal tuning region is as difficult to predict as for $\delta_{\alpha_{3}}$. Interestingly, $\delta_{\alpha_{3}}$ and $\delta_{\beta_{3}}$ have consistently an opposite sign, which is confirmed by plotting their ratio in Fig. 5. Since $\delta_{\alpha_{3}} / \delta_{\beta_{3}} \approx-0.05$ close to $\gamma_{\text {opt }}$, $\delta_{\alpha_{3}} \alpha_{3}+\delta_{\beta_{3}} \beta_{3} \approx 0$ if $\beta_{3} \approx 0.05 \alpha_{3}$. In other words, the potentially detrimental effect of the structural nonlinearity of the VdPD on the bifurcation behavior can be compensated through a proper design of the NLTVA's nonlinearity. Unlike the LTVA, the NLTVA can therefore be designed to enforce supercritical bifurcations in the optimal tuning region. At this stage, we provide a further justification of the cubic nonlinearity of the NLTVA. For a quadratic spring in the NLTVA, Eq. (9) would comprise the term $\delta_{\beta_{2}} \beta_{2}^{2}$ instead of $\delta_{\beta_{3}} \beta_{3}$. The compensation effect would not be achievable, because the sign of $\delta_{\beta_{2}} \beta_{2}^{2}$ could not be influenced by $\beta_{2}$.

\subsection{Two intersecting single Hopf bifurcations}

Although the investigation of the double Hopf bifurcation that occurs along the green line in Fig. 2(b) is beyond the scope of this paper, the separate analysis of the two intersecting single Hopf bifurcations gives already some insight into the dynamics. The eigenvalues of $\mathbf{W}$ at point $C$ are $\lambda_{1,2}= \pm j$ and $\lambda_{3,4}= \pm j / \sqrt{1+\varepsilon}$. By performing the analysis outlined in the previous section, first considering $\lambda_{1,2}$ as the critical eigenvalues and then $\lambda_{3,4}$, we obtain respectively

$$
\begin{aligned}
\delta_{1,2} & =\frac{1}{8}\left(-\frac{\varepsilon \sqrt{\varepsilon}}{1+\varepsilon}+\frac{3 \sqrt{\varepsilon}}{1+\varepsilon} \alpha_{3}-\frac{3(1+\varepsilon)}{\sqrt{\varepsilon}} \beta_{3}\right) \\
\delta_{3,4} & =\frac{3}{8}\left(-\sqrt{\varepsilon} \alpha_{3}+\frac{(1+\varepsilon)^{2}}{\sqrt{\varepsilon}} \beta_{3}\right) .
\end{aligned}
$$

The remarkable feature of Eqs. (10) and (11) is that the ratio between $\delta_{\alpha_{3}}$ and $\delta_{\beta_{3}}$ is $\delta_{\alpha_{3}} / \delta_{\beta_{3}}=-\varepsilon /(1+\varepsilon)^{2}$ and it is constant. 

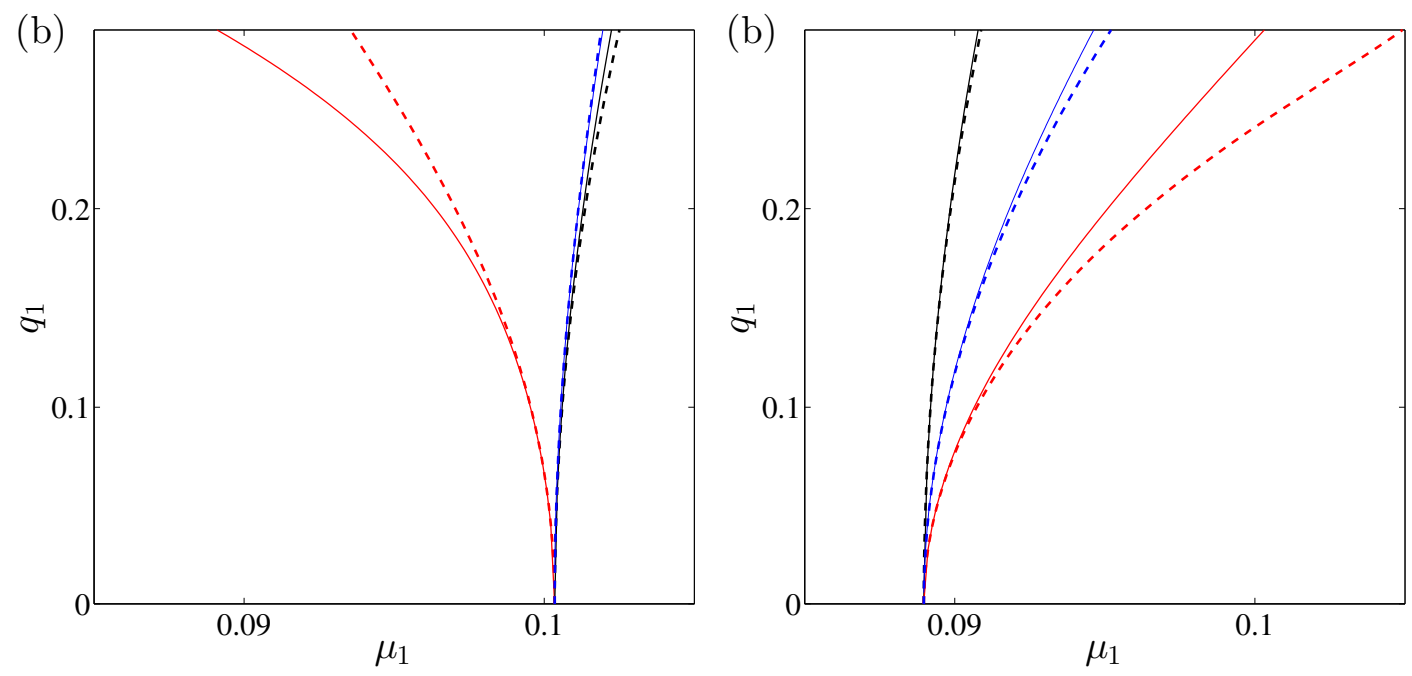

Figure 6: Bifurcation diagrams for $\mu_{2}=0.12$ and $\varepsilon=0.05$. (a) $\gamma=0.970$ (a), (b) $\gamma=0.985$. Black: VdP+LTVA ( $\alpha_{3}=0$ and $\left.\beta_{3}=0\right)$; red: VdPD+LTVA $\left(\alpha_{3}=0.3\right.$ and $\left.\beta_{3}=0\right)$; blue: VdPD+NLTVA $\left(\alpha_{3}=0.3\right.$ and $\left.\beta_{3}=0.0136\right)$. Thick dashed lines: numerical results (MATCONT); thin solid lines: analytical results.

The important practical consequence of this result is that the effect of $\alpha_{3}$ can be locally entirely compensated by $\beta_{3}=\varepsilon /(1+\varepsilon)^{2} \alpha_{3}$. It can be shown in fact that this ratio is constant along the whole line of double Hopf bifurcations. We also note that this formula is in excellent agreement with the expression $\delta_{\alpha_{3}} / \delta_{\beta_{3}} \approx-0.05$ obtained in the single Hopf case for $\varepsilon=0.05$.

\subsection{Proposed tuning rule for the nonlinear coefficient of the NLTVA}

According to the analysis in the previous sections, a fundamental result of this paper is that a properly-tuned NLTVA can annihilate the effect of the structural nonlinearity of the VdPD. Even though it is strictly valid along the line of double Hopf bifurcations, the tuning rule proposed herein for the nonlinear coefficient is

$$
\beta_{3}=\varepsilon /(1+\varepsilon)^{2} \alpha_{3}
$$

We anticipate that this rule is also valid in regions of single Hopf bifurcations, which is verified in this section.

The bifurcation diagrams predicted using the analytical developments of Section 4.1 and the numerical continuation software MATCONT ${ }^{[37]}$ are depicted in Fig. 6. Slightly detuned linear parameters, i.e., $\mu_{2}=0.12, \gamma=0.97 / 0.985$, are considered to show the robustness of our findings. Loss of stability occurs through a single Hopf bifurcation for the two parameter sets. Fig. 6 presents an excellent qualitative agreement between the analytical and numerical curves; the quantitative differences observed at higher values of $q_{1}$ are due to the fact that the analytical results are only valid locally. When there is no structural nonlinearity $\left(\alpha_{3}=0\right.$ and $\beta_{3}=0$ ), the bifurcation remains supercritical, and the LTVA works effectively on the classical VdP oscillator. The introduction of the structural nonlinearity $\left(\alpha_{3}=0.3\right)$ in the $\mathrm{VdP}$ oscillator gives rise to a subcritical or supercritical bifurcation in Figs. $6(\mathrm{a})$ and (b), respectively. This result confirms the difficulty to predict the bifurcation behavior of the coupled VdPD and LTVA system in the optimal region; it also highlights the detrimental role played by the structural nonlinearity of the VdPD oscillator. Conversely, the introduction of nonlinearity in the absorber $\left(\beta_{3}=0.0136\right)$ allows to guarantee a supercritical bifurcation, as for the system without nonlinearity. The compensation effect brought by the NLTVA is therefore clearly demonstrated.

For a more global perspective, Figs. $7(\mathrm{a})$ and (b) display the maximal value of $\alpha_{3}$ below which supercritical bifurcations for the LTVA are encountered. To avoid very large values in the vicinity of double Hopf bifurcations, the color map was trimmed at 1 . It is seen that the point $\mathrm{C}$ of optimal tuning of $\gamma$ and $\mu_{2}$ lies at the boundary between 0 and 1 , resulting in a design with virtually zero robustness. For positive (negative) values of $\alpha_{3}$, the solution for a robust absorber is to increase either $\gamma$ or $\mu_{2}$ (decrease $\gamma$ ), which necessarily results in an earlier LCO onset, i.e., $\mu_{1 \max }<\sqrt{\varepsilon} / 2$.

Figs. $7(\mathrm{c})$ and $(\mathrm{d})$ represent the same results for the NLTVA. For positive values of $\alpha_{3}$, point $\mathrm{C}$ now lies well inside the region 

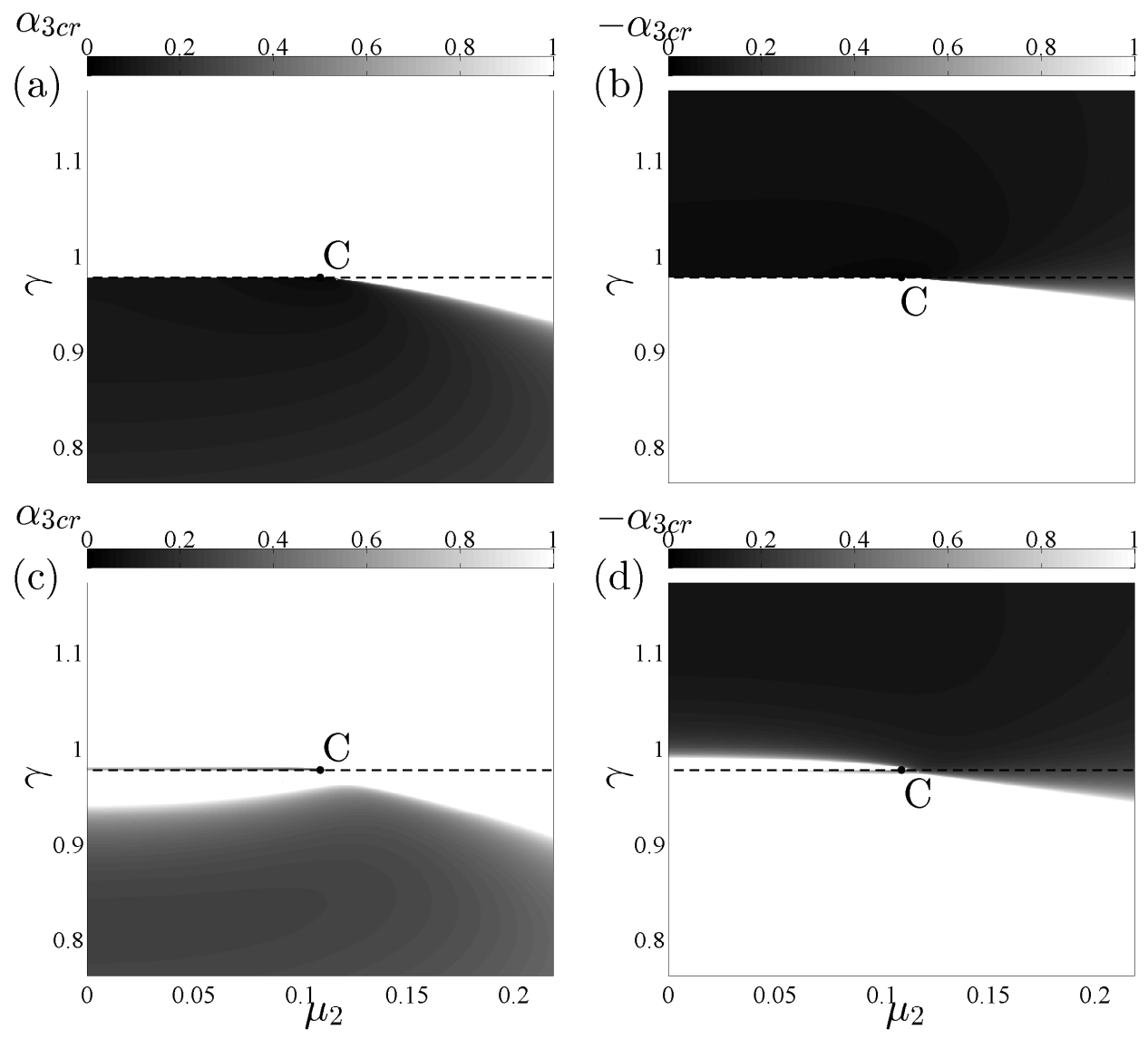

Figure 7: Maximal value of $\alpha_{3}$ below which supercritical bifurcations for the LTVA (a,b) and NLTVA (c,d) are guaranteed $\left(\varepsilon=0.05, \beta_{3}=0(\mathbf{a}, \mathbf{b})\right.$ and $\left.\beta_{3}=\varepsilon /(1+\varepsilon)^{2} \alpha_{3}\right)$. (a,c) Positive values of $\alpha_{3} ;(\mathbf{b}, \mathbf{d})$ negative values of $\alpha_{3}$. The dashed line corresponds to $\gamma=\gamma_{\mathrm{opt}}$ and the dot to $\mu_{2}=\mu_{2 \mathrm{opt}}$.
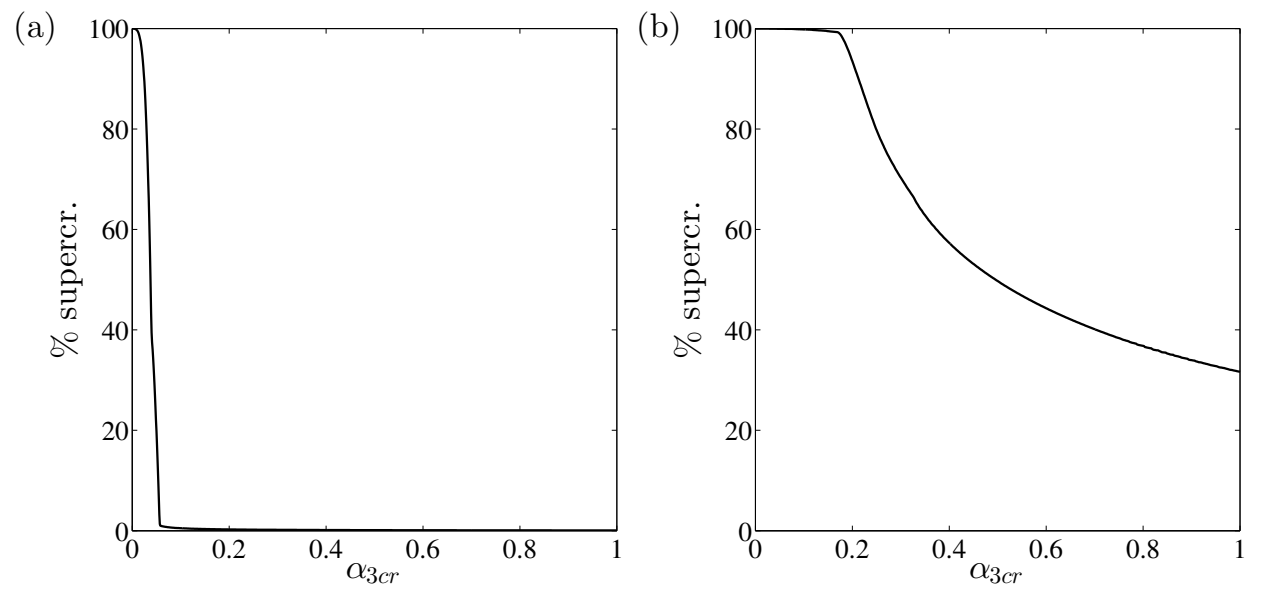

Figure 8: Probability to have a supercritical bifurcation for different values of $\alpha_{3}$ (in absolute value). (a) LTVA; (b) NLTVA. $\gamma$ and $\mu_{2}$ are within $\pm 1 \%$ and $\pm 5 \%$ of the corresponding optimum value, respectively.

where supercriticality is guaranteed, which clearly highlights the benefit of the NLTVA. For negative values of $\alpha_{3}$, the optimal point lies close to the boundary between 0 and 1, which means that there is much less margin for a robust design than for positive $\alpha_{3}$. 


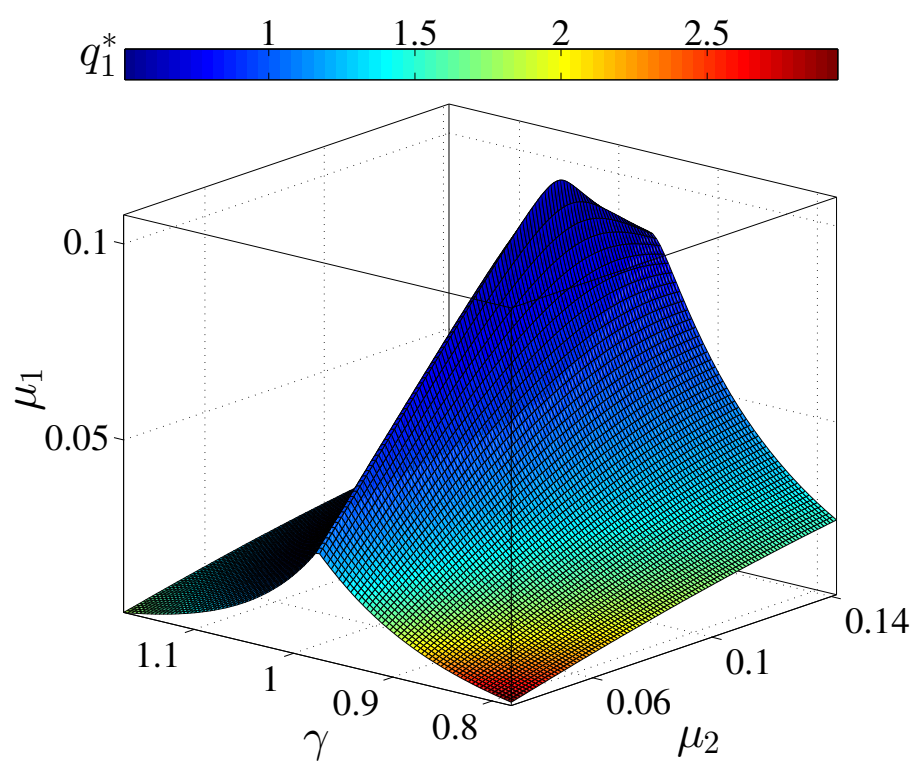

Figure 9: LCO amplitude along the stability boundary for $\alpha_{3}=0.08, \varepsilon=0.05, \beta_{3}=\varepsilon /(1+\varepsilon)^{2} \alpha_{3}=0.0036$ and

$$
\mu_{1}-\mu_{1 \mathrm{cr}}=0.01 \text {. }
$$

However, compared to the LTVA with negative $\alpha_{3}$, the NLTVA still possesses a larger region of supercritical behavior. Specifically, there is a new region $\gamma \approx \gamma_{\mathrm{opt}}$ and $\mu_{2}<\mu_{2 \mathrm{opt}}$ in which supercritically can be guaranteed.

Finally, Fig. 8 presents the probability to have a supercritical bifurcation as a function of $\alpha_{3}$ (in absolute value). To reflect a realistic design scenario, uncertainty of $\pm 1 \%$ and $\pm 5 \%$ on the values of $\gamma$ and $\mu_{2}$ around point $C$, respectively, are considered. Again, the superiority of the NLTVA over the LTVA is evident in these plots.

\section{REDUCTION OF THE AMPLITUDE OF LIMIT CYCLE OSCILLATIONS}

At this stage, the linear and nonlinear parameters of the NLTVA have been designed through stability (Section 3 ) and bifurcation (Section 4) analyses, respectively. There is therefore no much freedom left to mitigate the amplitudes of the LCOs in the postbifurcation regime.

\subsection{Local analysis}

Considering the normal form of a Hopf bifurcation, the amplitude of the generated LCOs is proportional to $\sqrt{-k_{1} / \delta}$, where $k_{1}$ is the real part of the eigenvalue related to the bifurcation (see Section 4.1). Because $k_{1}=0$ at the loss of stability, we consider its linear approximation, i.e., $\left.k_{1} \approx\left(\mathrm{d} k_{1} / \mathrm{d} \mu_{1}\right)\right|_{\mu_{1}=\mu_{1 c r}}\left(\mu_{1}-\mu_{1 \mathrm{cr}}\right)$. The LCO amplitude in the vicinity of the loss of stability is therefore

$$
r \approx \sqrt{-\left.\frac{\mathrm{d} k_{1}}{\mathrm{~d} \mu_{1}}\right|_{\mu_{1}=\mu_{1 \mathrm{cr}}} \frac{\mu_{1}-\mu_{1 \mathrm{cr}}}{\delta}}
$$

The maximal value of the LCO in physical space $\left(q_{1}^{*}\right)$, computed performing backward the transformation through which the normal form was obtained, is represented along the stability boundary in Fig. 9. It can be observed that the amplitude of the LCOs is minimized close to the optimal tuning region, which signifies that the design of the previous sections is also relevant for LCO mitigation. 

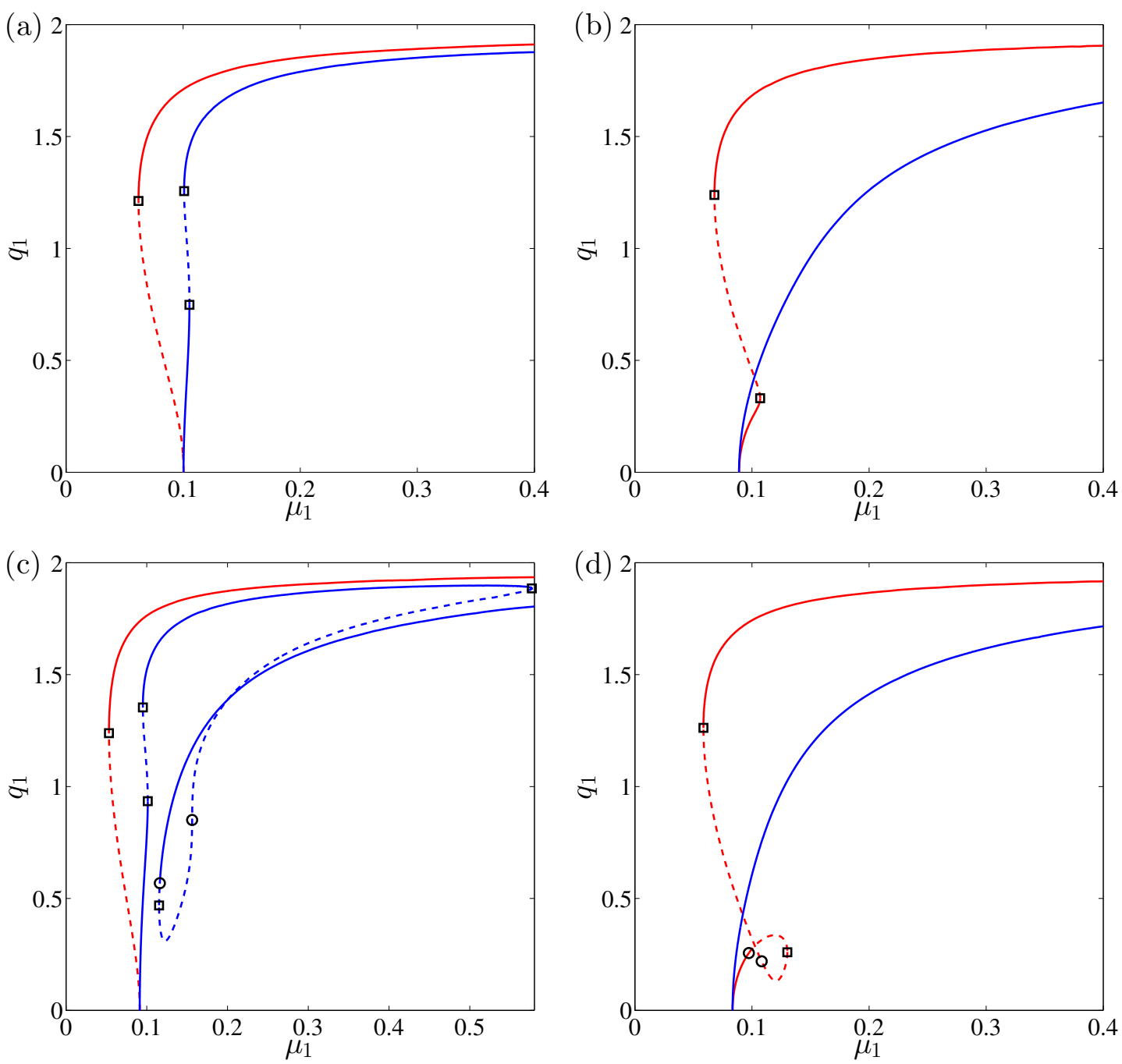

Figure 10: Bifurcation diagrams for $\mu_{2}=0.12$ (a,b) and $\mu_{2}=0.097 \mathbf{( c , d ) ,} \alpha_{3}=0.3$ and $\varepsilon=0.05$. (a,c) $\gamma=0.970,(\mathbf{b}, \mathbf{d})$ $\gamma=0.985$. Red: VdPD+LTVA $\left(\beta_{3}=0\right)$; blue: VdPD+NLTVA $\left(\beta_{3}=0.0136\right)$. Squares: fold bifurcations; circles: secondary Hopf bifurcations.

\subsection{Global analysis}

The previous analytical developments are valid only in the neighborhood of the bifurcation leading to LCO onset. The MATCONT software ${ }^{[37]}$ is now utilized to investigate large-amplitude LCOs.

Figs. 10(a) and (b) plot bifurcation diagrams for the case of a single Hopf bifurcation at the loss of stability. The same parameter values as those in Fig. 6 are used, but greater values of $q_{1}$ are investigated. A major difference with the local analysis is that fold bifurcations that can turn supercritical behavior into subcritical behavior are now encountered, which confirms the importance of global analysis. If the pair of folds that appears for the NLTVA in Fig. 10(a) cannot be considered as particularly detrimental, this is not the case for the LTVA in Fig. 10(b), where bistability in a significant portion of the stable region compromises the robustness of the linear absorber.

Figs. $10(\mathrm{c})$ and (d) represent the same results for a lower value of $\mu_{2}$ for which a double Hopf bifurcation is expected. The bifurcation diagrams are more complex with secondary Hopf (or Neimark-Sacker) bifurcations observed both for the LTVA and NLTVA; their analysis is beyond the scope of this paper. Apart from these new bifurcations, we note that the general trend of the curves is similar to that in Fig. 10, demonstrating a certain robustness of the absorbers with respect to parameter variations. 

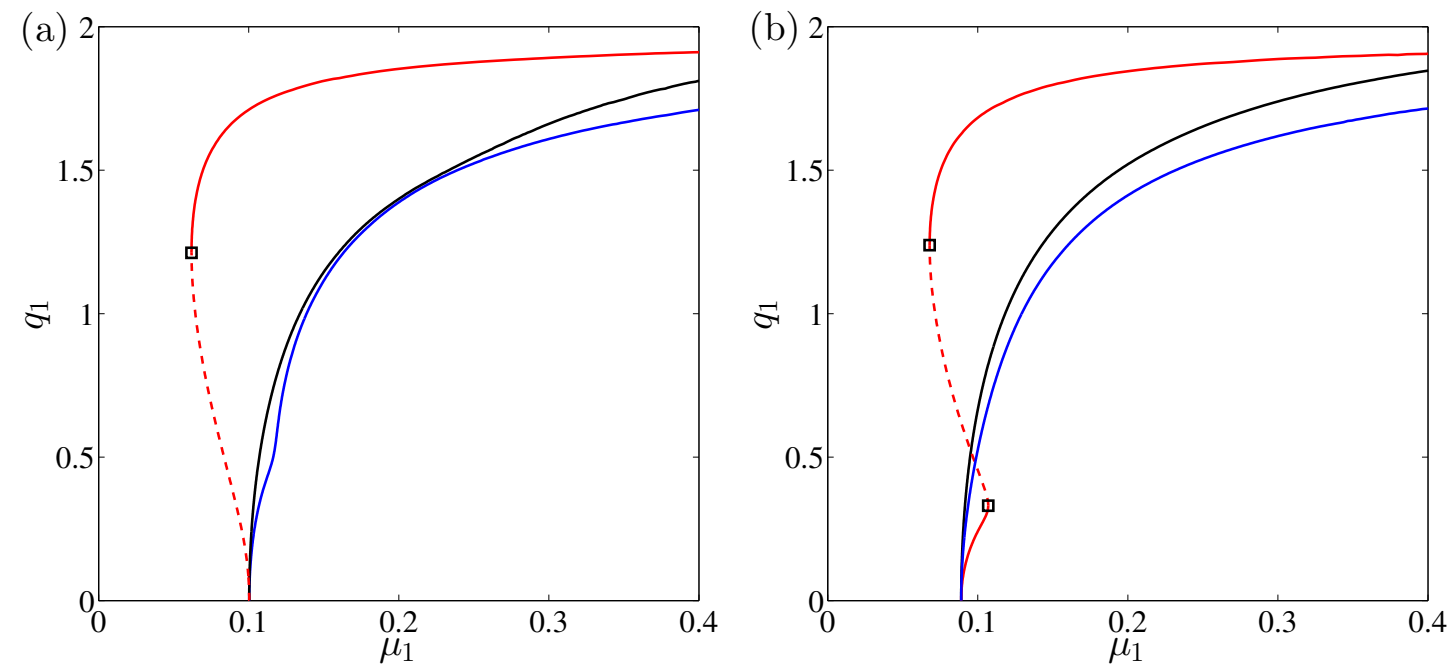

Figure 11: Bifurcation diagrams for $\mu_{2}=0.12$ and $\varepsilon=0.05$. (a) $\gamma=0.970$, (b) $\gamma=0.985$. Black: VdP+LTVA ( $\alpha_{3}=0$ and $\left.\beta_{3}=0\right)$; Red: VdPD+LTVA $\left(\alpha_{3}=0.3\right.$ and $\left.\beta_{3}=0\right)$; blue: VdPD+NLTVA $\left(\alpha_{3}=0.3\right.$ and $\left.\beta_{3}=0.018\right)$. Squares: fold bifurcations.

Fig. 11 considers again the single Hopf case, but with a slightly greater value of the nonlinear coefficient of the NLTVA, i.e., $\beta_{3}=0.018$ instead of 0.0136 . The NLTVA clearly outperforms the LTVA: not only LCO amplitudes are significantly smaller, but there is the complete absence of dangerous bistable regions. Another important result is the strong resemblance between the behaviors of the VdP+LTVA and VdPD+NLTVA systems. This suggests that the compensation of the nonlinearity of the VdPD by the nonlinearity of the NLTVA, which was observed in previous sections, is also valid at larger amplitudes.

\section{CONCLUSIONS}

The purpose of this paper was to investigate the performance of the NLTVA for suppression of self-excited oscillations of mechanical systems. A distinct advantage of this absorber is the complementary roles played by the linear and nonlinear springs.

Thanks to detailed stability and bifurcation analyses, a complete analytical design of the NLTVA, i.e., $\gamma=1 / \sqrt{1+\varepsilon}, \mu_{2}=$ $\sqrt{\varepsilon} /(2 \sqrt{1+\varepsilon})$ and $\beta_{3}=\varepsilon /(1+\varepsilon)^{2} \alpha_{3}$, was obtained. Although the double Hopf bifurcation scenario was not investigated herein, the preference should still be given to loss of stability through single Hopf bifurcations, which gives rise to simpler dynamics. This scenario can be enforced by, e.g., a small increase in the absorber damping $\mu_{2}$. The numerical validation of the analytical predictions also highlighted the benefit of considering a slightly greater value of the nonlinear coefficient $\beta_{3}$ compared to that suggested by the analytical formula.

Eventually, the NLTVA was shown to be effective for LCO suppression and mitigation, as it maximizes the stability of the trivial equilibrium point, guarantees supercritical bifurcations and reduces the amplitude of the remaining LCOs. Another interesting feature of the NLTVA is that its nonlinearity can be tuned to annihilate the effects of the potentially detrimental nonlinearity of the primary system. This compensation mechanism is appealing and clearly deserves more attention in future studies.

\section{ACKNOWLEDGEMENTS}

The authors G. Habib and G. Kerschen would like to acknowledge the financial support of the European Union (ERC Starting Grant NoVib 307265) 


\section{References}

[1] Denegri Jr., C.M., Limit cycle oscillation flight test results of a fighter with external stores, Journal of Aircraft 37, 761-769, 2000 .

[2] Trickey, S.T. ,Virgin, L.N., Dowell, E.H., The stability of limit cycle oscillations in a nonlinear aeroelastic system, Proceedings of the Royal Society A 458, 2203-2226, 2002.

[3] Stepan, G., Modelling nonlinear regenerative effects in metal cutting, Philosophical Transactions of the Royal Society A 359, 739-757, 2001.

[4] Mann, B.P., Bayly, P.V., Davies, M.A., Halley, J.E., Limit cycles, bifurcations and accuracy of the milling process, Journal of Sound and Vibration 277, 31-48, 2004.

[5] von Wagner, U., Hochlenert, D., Hagedorn, P., Minimal models for disk brake squeal, Journal of Sound and Vibration 302, 527-539, 2007.

[6] Coudeyras, N., Sinou, J.J., Nacivet, S., A new treatment for predicting the self-excited vibrations of nonlinear systems with frictional interfaces: The constrained harmonic balance method with application to disc brake squeal, Journal of Sound and Vibration 379, 1175-1199, 2009.

[7] Depouhon, A., Detournay, E., Instability regimes and self-excited vibrations in deep drilling systems, Journal of Sound and Vibration 333, 2019-2039, 2014.

[8] Griffin, O.M., Skop, R.A., The vortex-excited resonant vibrations of circular cylinders, Journal of Sound and Vibration 31, 235-249, 1973

[9] Ko, J., Kurdila, A., Strganac, T., Nonlinear control of a prototypical wing section with torsional nonlinearity, Journal of Guidance, Control and Dynamics 20, 1181-1189, 1997.

[10] Papatheou, E., Tantaroudas, N.D., Da Ronch, A., Cooper, J. E., Mottershead, J.E., Active control for flutter suppression: an experimental investigation, Proceedings of the International Forum on Aeroelasticity and Structural Dynamics (IFASD), Bristol, UK, 2013.

[11] van Dijk, N.J.M., van de Wouw, N., Doppenberg, E.J.J., Oosterling, J.A.J., Nijmeijer, H., Robust active chatter control in the high-speed milling process, IEEE Transactions on Control Systems Technology 20, 901-917, 2012.

[12] Stepan, G., Retarded Dynamical Systems: Stability and Characteristic Functions, Longman, UK, 1989.

[13] Niculescu, S.I., Gu K., Advances in Time-Delay Systems, Springer-Verlag, Berlin, Germany, 2004.

[14] Habib, G., Rega, G., Stepan, G., Nonlinear bifurcation analysis of a single-DoF model of a robotic arm subject to digital position control, Journal of Computational Nonlinear Dynamics 8, 011009, 2012.

[15] Mansour, W.M., Quenching of limit cycles of a van der Pol oscillator, Journal of Sound and Vibration 25, 395-405, 1972.

[16] Tondl, A., Quenching of self-excited vibrations equilibrium aspects, Journal of Sound and Vibration 42, 251-260, 1975.

[17] Rowbottom, M.D., The optimization of mechanical dampers to control self-excited galloping oscillations, Journal of Sound and Vibration 75, 559-576, 1981.

[18] Fujino, Y., Abe, M., Design formulas for tuned mass dampers based on a perturbation technique, Earthquake Engineering and Structural Dynamics 22, 833-854, 1993.

[19] Natsiavas, S., Vibration absorbers for a class of self-excited mechanical systems, Journal of Applied Mechanics 60, 382-387, 1993.

[20] Gattulli, V., Di Fabio, F., Luongo, A., Simple and double Hopf bifurcations in aeroelastic oscillators with tuned mass dampers, Journal of the Franklin Institute 338, 187-201, 2001.

[21] Gattulli, V., Di Fabio, F., Luongo, A., One to one resonant double hopf bifurcation in aeroelastic oscillators with tuned mass dampers, Journal of Sound and Vibration 262, 201-217, 2003.

[22] Gattulli, V., Di Fabio, F., Luongo, A., Nonlinear tuned mass damper for self-excited oscillations, Wind and Structures 7 , 251-264, 2004. 
[23] Haxton, R.S., Barr, A.D.S., The autoparametric vibration absorber, Journal of Manufacturing Science and Engineering 94, 119-125, 1972.

[24] Vyas, A., Bajaj, A.K., Dynamics of autoparametric vibration absorbers using multiple pendulums, Journal of Sound and Vibration 246, 115-135, 2001.

[25] Lee, Y.S., Vakakis, A.F., Bergman, L.A., McFarland, D.M., Suppression of limit cycle oscillations in the Van der Pol oscillator by means of passive nonlinear energy sinks, Structural Control and Health Monitoring 13, 41-47, 2006.

[26] Gendelman, O.V., Bar, T., Bifurcations of self-excitation regimes in a Van der Pol oscillator with a nonlinear energy sink, Physica D 239, 220-229, 2010.

[27] Luongo, A., Zulli, D., Aeroelastic instability analysis of NES-controlled systems via a mixed multiple scale/harmonic balance method, Journal of Vibration and Control 20, 1985-1998, 2014.

[28] Lacarbonara, W., Cetraro, M., Flutter control of a lifting surface via visco-hysteretic vibration absorbers, International Journal of Aeronautical and Space Sciences 2, 331-345, 2011.

[29] Habib, G., Detroux, T., Viguié, R., Kerschen, G., Nonlinear generalization of Den Hartog's equal peak method, Mechanical Systems and Signal Processing, http://dx.doi.org/10.1016/j.ymssp.2014.08.009i, 2014.

[30] Guckenheimer, J., Holmes, P., Nonlinear Oscillations, Dynamical Systems and Bifurcations of Vectors Fields, Springer-Verlag, NY, 1983.

[31] Ebelin, W., Herzel, H., Richert, W., Schimansky-Geier, L., Influence of noise on Duffing-Van der Pol oscillators, Journal of Applied Mathematics and Mechanics 66, 141-146, 1986.

[32] Szemplinska-Stupnicka, W., Rudowski, J., The coexistence of periodic, almost periodic and chaotic attractors in the Van der Pol-Duffing oscillator, Journal of Sound and Vibration 199, 165-175, 1997.

[33] Xu, J., Chung K.W., Effects of time delayed position feedback on a van der Pol-Duffing oscillator, Physica D 180, 17-39, 2003.

[34] Ji, J.C., Hansen, C.H., Stability and dynamics of a controlled van der Pol-Duffing oscillator, Chaos Solitons and Fractals 28, 555-570, 2006.

[35] Li, X., Ji, J.C., Hansen, C.H., Tan, C., The response of a Duffing-van der Pol oscillator under delayed feedback control, Journal of Sound and Vibration 291, 644-655, 2006.

[36] Nayfeh, A.H., Balachandran, B., Applied Nonlinear Dynamics, Analytical, Computational, and Experimental Methods, Wiley, New York, 2007.

[37] Dhooge, A., Govaerts, W., Kuznetsov, Y.A., MATCONT: A MATLAB package for numerical bifurcation analysis of ODEs, Journal ACM Transactions on Mathematical Software 29, 141-164, 2003. 